\title{
A Method of Controlling the Effect of Resistance in the Link Circuit of the Thomson or Kelvin Double Bridge*
}

\author{
David Ramaley
}

(May 12, 1960)

\begin{abstract}
Circuitry of the double bridge is reviewed to emphasize the significance of the link circuit as a part of the bridge network. A simple analysis demonstrates the need of some equivalent or substitute for low link circuit resistance in situations where adequately low link circuit resistance cannot be achieved physically. An appropriate method of controlling the potential difference in the link circuit is shown to accomplish the same effect as an actual reduction of resistance in the link circuit. The simple circuit modifications required to utilize this method are explained. A step-by-step procedure is outlined for making measurements with bridges incorporating these modifications, and some typical examples are discussed illustrating the advantages realized.
\end{abstract}

\section{Introduction}

Double bridges of the Kelvin type are convenient not only for the measurement of low-resistance standards, conductivity samples, and components, but also for calibrating resistors incorporated as intrinsic parts of circuits. When using the double bridge for calibrating resistors permanently connected in circuits, it is sometimes not feasible to achieve an arrangement with a link circuit of sufficiently low resistance. For such situations the precision of measurement will be diminished if the usual procedure is followed in balancing the bridge.

The method outlined in this paper utilizes a supplementary power source and an auxiliary galvanometer to minimize the potential difference in the link circuit. ${ }^{1}$ The application of this method has been found to reduce substantially the effect of excessive link resistance, and its practicability has been demonstrated in several different situations.

Descriptions and uses of double bridges are given in references listed at the end of this paper.

\section{Review of Double-Bridge Fundamentals}

Figure 1 is a circuit commonly used in double bridges. $X$ and $S$ are four-terminal resistors; $X$ is the unknown and $S$ is a reference standard of known resistance. The link circuit is designated as $L$ and includes the resistance of the link itself and also the additional resistance in the circuit between the resistor branch points $m$ and $n$. $A$ and $B$ are the main ratio arms, and $a$ and $b$ are the auxiliary ratio arms. These same symbols are used also to refer to resistance values. The ratio arms are connected to the potential terminals of $X$ and $S$ by leads designated as $L_{A}, L_{B}, L_{a}$, and $L_{b}$. When these notations are used to indicate lead resistances, they include not only the resistances of the external

* Contribution from the Radio Standards Laboratory, National Bureau of Standards, Boulder, Colo.

1 This method was suggested by the late Dr. Frank Wenner of the National Bureau of Standards in 1940 .

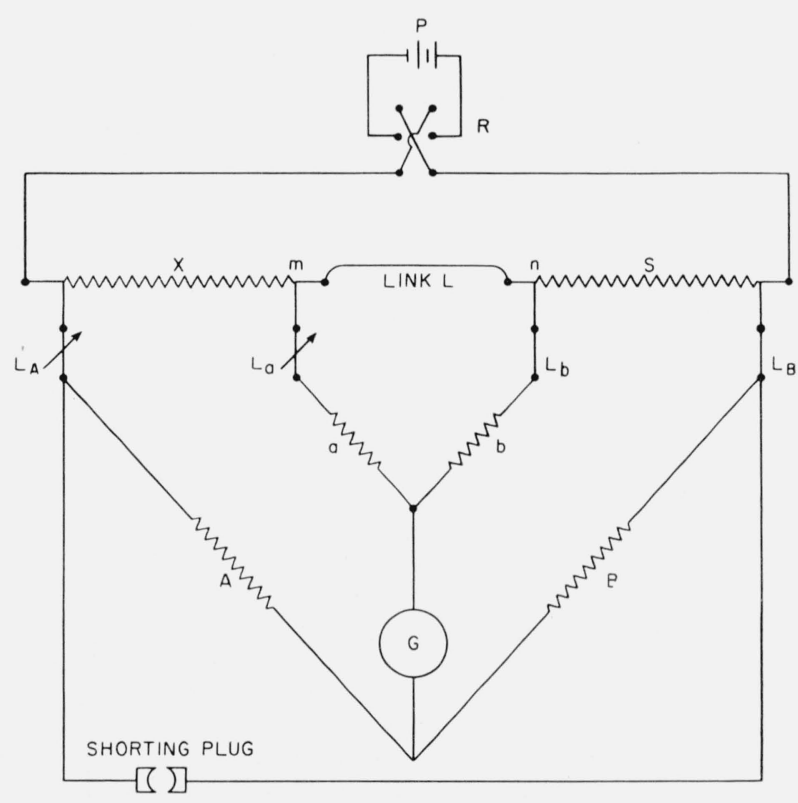

Figure 1. Double bridge.

connectors but all resistances between the ratio arms and the resistor branch points $m$, $n$, etc. Leads $L_{A}$ and $L_{a}$ are constructed to be conveniently varied in resistance by means of built-in adjustable rheostats. $P$ is a direct current power source such as a battery, $R$ is a reversing switch, and $G$ is a suitable galvanometer.

The delta network consisting of the two auxiliary ratio arms and the link circuit can be transformed readily into the equivalent $Y$ network. The resistance of that branch of the $Y$ between the $Y$ junction point and $m$, adjacent to the resistor, $X$, is $L\left(a+L_{a}\right) /\left(a+L_{a}+b+L_{b}+L\right)$. The resistance of that $Y$ branch, between the $Y$ junction and $n$, adjacent to the resistor, $S$, is $L\left(b+L_{b}\right) /\left(a+L_{a}+b+\right.$ $\left.L_{b}+L\right)$. The third $Y$ branch is in series with the 
galvanometer and need not be considered in the present discussion because it does not affect the position of bridge balance.

This transformation enables one to treat the double bridge as a simple Wheatstone arrangement, from which one obtains the equation of balance:

$$
\frac{A+L_{A}}{B+L_{B}}=\frac{X+\frac{L\left(a+L_{a}\right)}{\left(a+L_{a}+b+L_{b}+L\right)}}{S+\frac{L\left(b+L_{b}\right)}{\left(a+L_{a}+b+L_{b}+L\right)}} .
$$

When rearranged this equation becomes

$$
\begin{array}{r}
X=\frac{\left(A+L_{A}\right) S}{\left(B+L_{B}\right)}+\frac{L\left(A+L_{A}\right)\left(b+L_{b}\right)}{\left(B+L_{B}\right)\left(a+L_{a}+b+L_{b}+L\right)} \\
-\frac{L\left(a+L_{a}\right)}{\left(a+L_{a}+b+L_{b}+L\right)}
\end{array}
$$

If the substitions:

$$
\begin{aligned}
\frac{A+L_{A}}{B+L_{B}} & =\frac{A(1+g)}{B}, \\
\frac{a+L_{a}}{b+L_{b}} & =\frac{A(1+h)}{B},
\end{aligned}
$$

and

$$
\frac{L\left(b+L_{b}\right)}{S\left(a+L_{a}+b+L_{b}+L\right)}=D
$$

are made, eq (2) becomes

$$
X=\frac{S A[1+g+D(g-h)]}{B} .
$$

This can be considered a fundamental equation of the double bridge and can be reduced to the simple Wheatstone equation, $X=S A / B$, provided the terms $g$ and $D(g-h)$ can be made negligible. This is achieved in practice as follows: With $A$ and $B$ shorted out and with the bridge essentially balanced, the term, $g$, commonly is made very small by adjusting $L_{A}$ so that the leads $L_{A}$ and $L_{B}$ have nearly the same ratio as $X$ to $S$ or $A$ to $B$. The term, $h$, is made closely equal to $g$ by making $a / b$ as nearly as possible the same as $A / B$, not only by using a common set of controls for the main and auxiliary ratio arms but also by removing the link and then making a balance by adjusting the ratio $L_{a} / L_{b}$ to nearly $X / S$ or $A / B$. The term $D$ usually is much smaller than one and normally is minimized by using a link circuit of low resistance.

In some situations it is impractical to arrange a link circuit with suitably low resistance. Then the balancing procedure employed in minimizing the term $(g-h)$ must be accomplished with much higher precision to avoid deterioration in accuracy. This necessity is evident from the term $D(g-h)$ in eq (6). If $L$ is large, $D$ also is proportionately large, and the term $(g-h)$ must be made extremely small. However, this requires extremely fine adjustment of the leads, and some double bridges are not provided with means of making sufficiently precise lead adjustments, so that the accuracy of measurement is definitely limited. A reduction in accuracy under conditions of excessive link circuit resistance therefore may be unavoidable unless one makes some modification of the usual double bridge. Such a modification is described below. It minimizes the effect of link resistance even though the actual resistance of the link is abnormally high.

With the bridge in balance and power applied, the ratio of the potential difference $E_{L}$, developed across the link circuit, to the sum of the potential differences $E_{X}+E_{S}$, developed across the resistors $X$ and $S$, is given by

$$
\frac{E_{L}}{E_{X}+E_{S}}=\frac{\frac{L\left(a+L_{a}+b+L_{b}\right)}{\left(a+L_{a}+b+L_{b}+L\right)}}{X+S} .
$$

When all circuits of the bridge have been properly adjusted, the ratio of $S$ to $X$ is almost identical to the ratio of $B+L_{B}$ to $A+L_{A}$ and also to the ratio of the corresponding auxiliary branch arms. It may be assumed with sufficient accuracy that

$$
\frac{S}{X+S}=\frac{b+L_{b}}{a+L_{a}+b+L_{b}} .
$$

Combining eqs (7) and (8) gives

$$
\frac{E_{L}}{E_{X}+E_{S}}=\frac{L\left(b+L_{b}\right)}{S\left(a+L_{a}+b+L_{b}+L\right)}
$$

which from eq (5) is the term $D$. For practical use, then, the double bridge eq (6) may be written as

$$
X=\frac{S A}{B}\left[1+g+\frac{E_{L}(g-h)}{E_{X}+E_{S}}\right] .
$$

Consequently, reduction of the potential difference $E_{L}$ across the link circuit would produce the same effect as reduction of the link resistance and would eliminate the necessity of adjusting the ratios $L_{a}$ to $L_{b}$ and $L_{A}$ to $L_{B}$ with higher precision than ordinarily used when the link resistance is small. This can be accomplished by introducing into the link circuit a potential difference of such magnitude and polarity that the potentials at the branch points $m$ and $n$ are brought to practically the same value. This applied potential difference is introduced across an auxiliary resistor of appropriate magnitude which has been inserted into the link circuit.

\section{Double Bridge Modification for Abnormal Link Circuit Resistance}

Figure 2 is the circuit of the modified double bridge. $P^{\prime}$ is the auxiliary power supply. $Z$ is the resistor inserted into the link circuit across which the auxiliary potential difference is applied. $R$ and $R^{\prime}$ are reversing switches which can be operated simultaneously. $G^{\prime}$ is an auxiliary galvanometer 


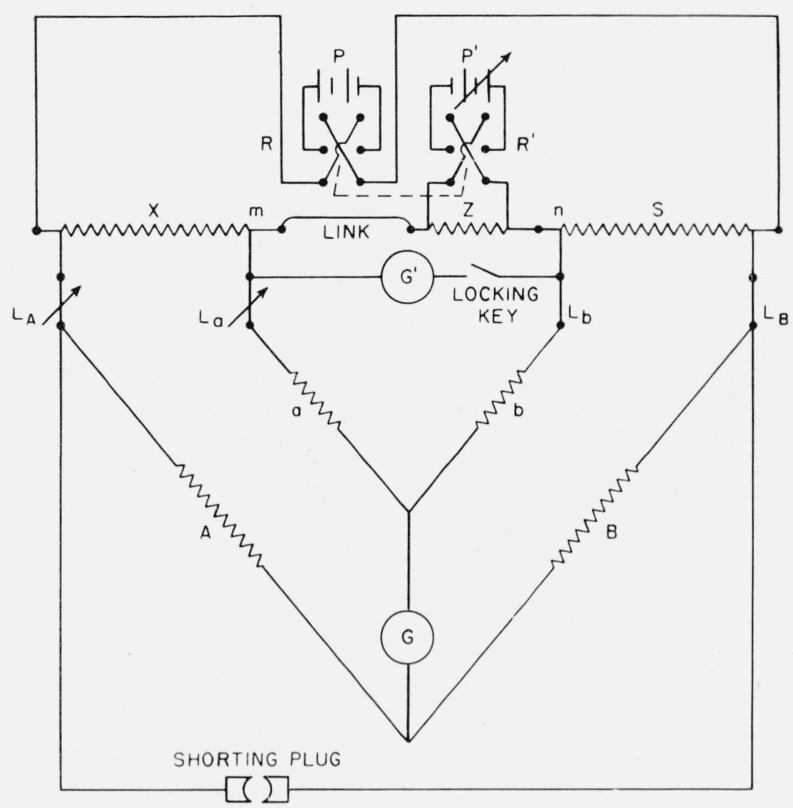

FIgURE 2. Double bridge with modified link circuit.

used in detecting potential differences in the link circuit. The auxiliary galvanometer is connected as shown rather than to $m$ and $n$ because of physical difficulties sometimes encountered in connecting directly to the branch points. By varying the potential difference applied to $Z$ from the auxiliary power source, the potentials at the branch points can be adjusted to practically the same value.

The modified double bridge can be balanced by the following steps:

(1) With both battery circuits closed, the potential difference indicated by the auxiliary galvanometer is minimized by varying $P^{\prime}$.

(2) A preliminary balance of the bridge is made by adjusting in unison the main and auxiliary ratio arms while operating the two reversing switches simultaneously until a balance is indicated by the main galvanometer.

(3) The leads in series with the main ratio arms are adjusted to the proper ratio by shorting out the main arms and adjusting one of these leads. Both power sources are used simultaneously, and the balance is indicated by the main galvanometer.

(4) With the main power supply disconnected, the lead resistances in series with the auxiliary ratio arms are adjusted to the proper ratio by varying one of the leads until balance is indicated by the main galvanometer. Current is supplied by the auxiliary power supply. An alternate method of adjustment, commonly followed for double bridge operation, is to open the link circuit and use only the main power supply while adjusting the leads to the auxiliary ratio arms.

(5) Final balance is achieved by repeating step 2 . If the bridge is too far out of balance at the outset, a repetition of steps 3, 4, and 2 may be required.
The readings of the bridge ratio arms $A$ and $B$ corresponding to the final balance are used to compute the value of the resistor $X$.

Except for step 1, involving the auxiliary galvanometer, the above procedure is that commonly used in balancing a double bridge. The selection of circuit components as well as the precision necessary in making the adjustments in steps 1 to 5 will be governed by the particular case under consideration and the desired accuracy of measurement. Ordinarily step 1 is not unduly critical. However, good stable power supplies and switches are required, since variations in either power source adversely affect the accuracy obtainable with the modified double bridge.

This modification was developed in connection with the calibration of a special microvolt potentiometer. The measuring circuit of this potentiometer utilizes low-resistance elements connected in series within a thermally shielded enclosure. In the measurement of one of these resistors using a double bridge, current lead connections were found to be feasible only by incorporating the adjacent, permanently connected, circuit resistors. This arrangement produced an abnormally high link circuit resistance, and the resulting ratio of the link circuit resistance to the sum of the resistances of $X$ and $S$ was approximately 10 . With the modified double bridge, however, it was feasible to measure the low-resistance element to the desired accuracy.

\section{Examples}

The improvement in accuracy achieved by use of this modification can be evaluated for each individual case. It depends upon the resistors under comparison and upon the bridge parameters. Both the main and auxiliary ratio arms commonly have resistances of 50 to 500 ohms. Lead resistances commonly range in magnitude from 0.01 to possibly 2 ohms. Resistors suitable for comparison in a double bridge may range in magnitude from a few microhms to about an ohm.

An arrangement that will serve as an example for this ordinary range of measurements will now be considered. Let us assume that four-terminal 0.001 ohm resistors $X$ and $S$ are to be compared in a bridge with ratio arms of approximately $100 \mathrm{ohms}$ and lead resistances of about $1 \mathrm{ohm}$, and that the ratio of $A$ to $B$ can be adjusted to or estimated to about one part in a million. The accuracy of adjustment of the resistor $S$ and the ratio of $A$ to $B$ will be considered to be known adequately. The uncertainty associated with these terms outside of the bracket in eq (6) is a systematic error inherent in the measurement regardless of the arrangement of the link circuit or lead connections and is not relevant to the present consideration. We are concerned primarily with the effect of link resistance and the precision of lead balances upon the correction terms $g$ and $D(g-h)$ in eq (6). Let us assume that a precision of $\pm 30 \mathrm{ppm}$ is desired and that the expression $S A / B$ can be treated as a constant during the adjust- 
ments. The sum of the correction terms $q$ and $D(g-h)$ in eq (6) can differ with each successive measurement depending upon how consistently balances are executed but in no case should exceed \pm 0.00003 at the time of reading if the desired precision is to be maintained. Ordinarily a very lowresistance shorting link can be used and the term $D$ in eq (5) or its equivalent $E_{L} /\left(E_{X}+E_{S}\right)$ or $L /(X+S)$ is less than or equal to one. To maintain the desired precision for this situation, $g$ and $h$ each can be as large as \pm 0.00001 without giving $g+D(g-h)$ a value larger than \pm 0.00003 . The ratio arms including the leads need not be balanced more precisely than \pm 10 parts in a million or $\pm 0.001 \mathrm{ohm}$. This implies that the leads must be adjusted to one part in a thousand because they are $1 \mathrm{ohm}$ in magnitude. If this is accomplished, each of the terms $g$ and $h$ in eqs (3) and (4), will be not larger than \pm 0.00001 and the sum of $g$ and $D(g-h)$ will be not larger than \pm 0.00003 .

Next consider the situation in which it is impossible to arrange a link circuit so low in resistance that $D$ can be held to a value of one or less. The expression $D(g-h)$ can be held within the prescribed limit only by adjusting the leads more closely than $\pm 0.001 \mathrm{ohm}$ because $(g-h)$ must be decreased to the same extent that $D$ is increased. Contact resistance variations in adjustable lead circuits commonly exceed $0.0001 \mathrm{ohm}$ and thus may limit the fineness of lead adjustment. Furthermore, some rheostats used for the adjustable leads may not be variable in steps finer than 0.001 ohm. If we assume such excellent conditions that variations in contact resistances of $\pm 0.0001 \mathrm{ohm}$ constitute the factor limiting the fineness of adjustment of leads, $g$ and $h$ in eqs (3) and (4), can be reduced to \pm 0.000001 and $(g-h)$ to a maximum of \pm 0.000002 . For these values $D$ must be smaller than 15 if diminished precision is to be avoided.
For $D=15, g+D(g-h)$ could be a maximum of $\pm 0.000001 \pm 15( \pm 0.000001 \pm 0.000001)$ or \pm 0.000031 which is slightly larger than the permissible $\pm 0.00003$

If in the comparison of the above 0.001-ohm resistors, the value of $L$ cannot be physically reduced below 0.05 ohm, $D$ will be about 25 , as computed from eq (5). The terms $g+D(g-h)$ will be equal to \pm 0.000051 if the leads can be adjusted to \pm 0.0001 ohm and to \pm 0.00051 if the leads can be adjusted to $\pm 0.001 \mathrm{ohm}$. If the link potential difference is reduced by a factor of 25 , then for making a measurement, the conditions are the same as if $D$ were actually no greater than one. In considering again the favorable condition in which the leads can be adjusted to $0.0001 \mathrm{ohm}$, the improvement in precision obtained by reducing the link potential will be in the ratio of 0.000051 to 0.000031 or a factor of about two. In the case of the bridge in the less favorable condition, the precision gained will be about 0.00051 to 0.00003 or about 17 . These same factors likewise can be regarded as representing an unavoidable loss in precision if this modification is not employed. Other examples with different circuit values and requirements can be treated in like manner.

\section{References}

[1] F. Wenner, The four-terminal conductor and the Thomson Bridge, Bul. BS 8, 559 (1912) S181.

[2] F. Wenner, Methods, apparatus, and procedures for the comparison of precision standard resistors, J. Research NBS 25, 229 (1940) RP1323.

[3] J. L. Thomas, Precision resistors and their measurement, NBS Circ. 470 (1948).

[4] F. K. Harris, Electrical measurements, p. 282-288.(John Wiley \& Sons, Inc., New York, N.Y., 1952).

(Paper 64C4-44) 